\title{
Birds of a feather
}

Matter as we know it - in fundamental physics, at least - is mostly a passive thing. The particles making up anything from liquid water to the ionized plasma in a star's interior don't generally act as creative agents, capable of producing and expending their own energy. They have law-like behaviour determined by external initial conditions or other constraints; in classical or quantum physics, particles and fields evolve without autonomy or self-action.

This is, normally, how we conceive of 'matter'. But substance or material needn't be so passive. Think of muscle tissue as a medium, and you see one with the capacity to contract on its own by using a store of internal energy. The inherent activity of this medium has important consequences. Normally, its dynamics organize the orderly muscle contractions that pump blood effectively. Equally, however, life-threatening episodes of ventricular tachycardia can arise from waves of contraction that cycle repeatedly around the heart in a closed path, disrupting normal heart behaviour.

Even more active is a colony of bacteria, each member of which can carry out sustained motion as well as complex signalling and coordination with others. Or think of a flock of birds or herd of migratory animals. Despite obvious differences, these systems all share a similar character as collectives of interacting and self-propelling elements with internal sources of energy. It's natural to think of all of these as examples of a more general kind of 'active' matter - a new frontier where ideas from physics on the principles of order and organization are proving very useful.

Take flocks, for example. One study a couple of years ago found that changes in the velocity of any one bird in a flock of starlings rapidly influences the velocity of all of the other birds; notably, the influence seems to propagate in an undamped way across the flock, reaching all birds very effectively with minimal dissolution of the signal

(A. Cavagna et al., Proc. Natl. Acad. Sci. USA 107, 11865-11870; 2010). This is interesting, as it suggests some kind of evolutionary tuning of interactions to produce optimal sensitivity in the group's ability to respond to signals gathered by any one member. But there's a deep analogy to physics too.

Indeed, the interactions between birds seem to be organized in a 'scale-free' way,

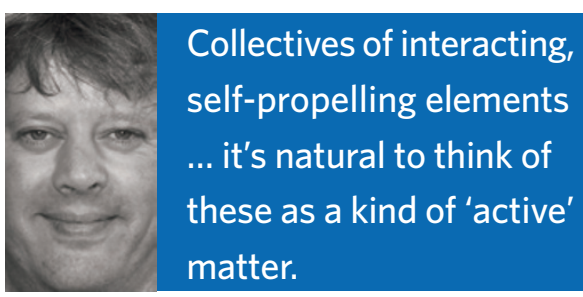

with the flock's sensitive dynamics closely linked to the behaviour of a physical substance held near a critical point between an organized and disorganized phase. This gives each bird, in effect, a range of perception extending out far beyond its nearest neighbours and limited only by the size of the flock itself.

The physical analogies for flocks, colonies and other active-matter systems go much further. Indeed, theorists have been able to develop the rudiments of a natural 'hydrodynamics' of such systems, closely akin to the familiar hydrodynamics of ordinary fluids. Such models can be derived either from basic symmetry considerations or by coarse-graining microscopic models based on elementary interactions, and can yield basic equations for the evolution of variables such as the density and orientational order. The resulting equations, which ought to apply even to living organisms, make some strong predictions.

The first is that order and organization should be ubiquitous in such systems, at least in the absence of too much disrupting noise. Of course, we do see order everywhere in animal herds, in bacterial colonies, even in the cell cytoskeleton, a complex network of protein filaments that provides a cell with its elastic and viscous properties. The latter is a solution or gel made of polymers, but one that is highly active - undergoing continuous dynamic reorganization on the basis of a selforganizing biochemistry.

Admittedly, the simplest theory offers only a crude start, as it suggests a rather monolithic order with all elements locked into identical behaviour, unlike order mixed with disorder as we see in nature. But that is the ideal theory, and a little further investigation shows something else - that the uniformly ordered state should actually be unstable against the growth of waves at long wavelengths, and should soon tend to fall apart into a kind of weak turbulence with orientation and motion only coherent over short distances. This is precisely what happens in fairly dense animal herds, and has been confirmed in some detail in studies with bacteria.

The hydrodynamics of flocks (in the general sense) shows more as well - for instance, an unusually pronounced growth of fluctuations with the number of elements in the system. In traditional physics, the usual arguments from statistics and thermodynamics suggest that variations in the bulk properties of a substance should grow smaller in proportion to $1 / \sqrt{ } N$, if $N$ is the number of particles. Hence, large systems have highly stable and well-defined bulk properties, such as pressure or density. For active matter, the result is in contrast a much slower decrease with increasing $N$, making giant fluctuations on a scale comparable to the whole system much more likely. From this point of view, the behaviour of bacterial colonies, the spatial structure of which vacillates wildly on an ordinary basis, does not look at all surprising even in the absence of possible behavioural explanations. Indeed, careful measurements with bacterial colonies confirm the theoretical predictions accurately.

Of course, the real pay-off from understanding the basic physics of active matter will be to give scientists a platform for building practical insight into systems of biological, engineering or medical interest (a nice review is M. C. Marchetti et al., Rev. Mod. Phys.; in the press). Experiments have observed giant fluctuation in the cellular cytoskeleton, yet the origin of such dynamics remains a mystery. They could have no functional role - merely being physics. Or they could be the result of clever harnessing of physical modes by the cellular regulatory apparatus, an exploitation of the possibilities of active matter long before physicists knew of them. Most probably the real dynamics will be a complex mix of both functional and non-functional dynamics, and a deeper theory of active matter will be required to tease them apart.

In this case, going further will require that theories include mechanisms for the exchange of richer interactions purposive signals - between elements. Such work has not yet really got off the ground, but it is clear that a truly deep understanding of biology will have to take physics of a wide range of more active materials into account.

\section{MARK BUCHANAN}

